\title{
Aprendizaje basado en problemas en matemáticas: el concepto de fracción
}

\section{Learning based on mathematical problems: the fraction concept}

Deisy Marieta Cristancho Cárdenas*
Leidy Yalima Cristancho Cárdenas**

Fecha de recepción: 13 de diciembre de 2016

Fecha de aceptación: 2 de julio de 2017

Artículo de reflexión

\section{Resumen}

El presente artículo muestra diferentes aspectos sobre la intervención realizada en la Institución Educativa Politécnico Álvaro González Santana, con base en experiencias anteriores en las cuales se identificó la estrategia didáctica como elemento fundamental en el proceso de aprendizaje de la fracción. Además, se consideraron resultados de investigaciones sobre la implementación del ABP en la enseñanza de las matemáticas; hilados con teorías amparadas en el constructivismo. En este proceso, el aprendizaje surgió como efecto de la planeación e implementación de situaciones problema, enmarcadas en un proceso de investigación cualitativa que permite a los docentes de matemáticas, en séptimo grado, reconocer los efectos de la utilización de la metodología ABP para desarrollar competencias como la resolución de problemas, y que estas se concreten con el desarrollo del pensamiento matemático involucrando la fracción y sus significados.

Palabras clave: educación, estrategia didáctica, enseñanza, aprendizaje, problemas.
* Institución Educativa Politécnico Álvaro González -Boyacá, Colombia deisycriscar@hotmail.com ** Institución Educativa

Politécnico Álvaro González - Boyacá, Colombia leidycris21@hotmail.com

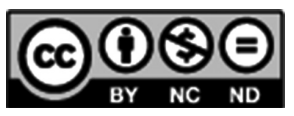




\section{Abstract}

This article shows different aspects of the intervention carried out at the Educational Institution "Álvaro González Santana”, based on previous experiences in which the didactic strategy was identified as a fundamental element in the learning process of the fraction. In addition, research results on the implementation of ABP Methodology in the teaching of mathematics were considered; Spins with theories covered in constructivism.
In this process, learning arose as an effect of the planning and implementation of problem situations, framed in a qualitative research process that allows math teachers, in seventh grade, to recognize the effects of using the $\mathrm{ABP}$ methodology to develop competences like the resolution of problems, and that these are materialized with the development of mathematical thinking involving the fraction and its meanings.

Keywords: education, didactic strategy, teaching, learning, problems. 


\section{Introducción}

El concepto de fracción se desarrolla desde los grados de primaria, y se afianza en grado séptimo, dado que allí se relaciona con diferentes formas de aplicación. No obstante, al revisar el uso de este concepto en estudiantes de diferentes grados, se identifican diferentes vacíos, fundamentalmente en la construcción de conocimiento desde los saberes previos y el vínculo del concepto con el contexto, lo que a los estudiantes les dificulta la interpretación de las relaciones entre el concepto y sus significados (Streefland, 1991). Teniendo en cuenta esos vacíos, las investigadoras realizaron una intervención con estudiantes de séptimo grado de la Institución Educativa Politécnico Álvaro González Santana (IEPAGS), cuyo propósito fue implementar una estrategia didáctica como el Aprendizaje Basado en Problemas (ABP) enfocada en el aprendizaje activo, donde el estudiante aprende desde situaciones problema que emergen de los contenidos de la asignatura y le permiten llegar a generalizar la relación entre los significados de la fracción y, a su vez, particularizar el concepto en otras situaciones en contexto (Obando Zapata \& Muñera Córdoba, 2003). Todo ello, para desarrollar en el estudiante competencias matemáticas (actitudes y habilidades del estudiante) y el pensamiento matemático desde los significados de la fracción, de acuerdo con lo establecido por el Ministerio de Educación Nacional (MEN, 2016) en los estándares de competencias matemáticas.

\section{Antecedentes}

A continuación, se presentan algunos estudios relacionados con la resolución de situaciones problema y/o el aprendizaje de la fracción en sus diferentes representaciones:

En el estudio de Castaño (2014), se manifestó la necesidad de buscar alternativas diferentes a las tradicionales de acuerdo con los resultados obtenidos para la categoría dificultades didácticas. Estas dificultades, desde la perspectiva del docente, requieren una búsqueda de estrategias, en la que emerjan categorías como la utilización de la realidad y la perspectiva del estudiante de acuerdo con los modelos de procesamiento de información, haciendo énfasis en las ventajas del aprendizaje contextualizado que involucra la construcción del conocimiento, con los saberes previos del estudiante y la relación con el entorno.

En ese sentido, Escolano \& Gairín (2005) hacen referencia a las consecuencias de la práctica docente, y a la dificultad en la comprensión debido a una aparente facilidad del docente al presentar el concepto de número racional como relación parte-todo, haciendo referencia a obstáculos presentes en la construcción significativa de las fracciones.

De acuerdo con lo anterior, Morales Díaz (2014) reconoce los errores y dificultades que presentan los estudiantes en la resolución de problemas con números fraccionarios empleando como contexto el ambiente natural del aula, como los aspectos conceptuales relacionados con la noción parte-todo en la solución de problemas con fraccionarios, resaltando el sobre uso de dicha noción, en especial en la solución de problemas que requieren la aplicación del número fraccionario.
En el estudio de Castaño (2014), se manifestó la necesidad de buscar alternativas diferentes a las tradicionales de acuerdo con los resultados obtenidos para la categoría dificultades didácticas. 
Por su parte, Díaz (2010) estima que no es lo mismo hacer un ejercicio que resolver un problema, ya que en el primer caso se aplica un algoritmo, mientras que en el segundo el estudiante

debe dar una explicación con respuestas coherentes a las condiciones que plantea, llegando a ser consciente de la responsabilidad que asume en el proceso de enseñanzaaprendizaje (Caseida \& Dávila, 2006).
Por otro lado, la investigación realizada por Silva Laya \& Rodriguez (2011), identificó la importancia del trabajo colaborativo como puerta hacia la búsqueda de soluciones presentadas desde distintos puntos de vista, por lo que la evaluación individual se convierte en cíclica de acuerdo con la teoría constructivista desde el error o el acierto del estudiante, una vez que retorna a la solución desde la socialización.

Al respecto, Fuenlabrada et al. (2005) reafirman la importancia de permitir a los niños ensayar formas novedosas y particulares de resolver problemas más allá de los métodos convencionales; los alumnos, conacercamientos más creativos, podrían socializar sus procedimientos y colaborar con sus compañeros en el desarrollo de estrategias, explotando así la zona de desarrollo próximo sugerida por Vygotsky.

Así mismo, Perera \& Valdemoros (2009) mostraron que se favoreció la consolidación de la noción de fracción y de algunos de sus significados (relación parte-todo, medida, cociente intuitivo y rudimentos de operador multiplicativo) en cuarto grado de educación primaria. Todo esto, a través de la resolución de situaciones problemáticas de la vida real, planteadas en el programa de enseñanza, en las que el niño reconstruyó mentalmente sus experiencias cotidianas en un ambiente de interacción donde prevalecieron actitudes lúdicas durante el desarrollo de la enseñanza experimental de las fracciones.

$\mathrm{Al}$ respecto de la metodología utilizada, Angulo \& Castaño (2011) contrastaron el uso de varias estrategias metodológicas frente a la tradicional, resaltando las ventajas de cada una de ellas, especialmente en la estrategia didáctica ABP, ya que mejora el proceso enseñanza-aprendizaje, debido a las diversas formas de trabajo que propone, donde el estudiante interactúa con el conocimiento y lo potencia, encontrando el sentido de la temática que está aprendiendo y su importancia, mientras que el docente interactúa con los estudiantes actuando como guía, facilitando el proceso enseñanzaaprendizaje de las matemáticas.

Por su parte, Díaz (2010) estima que no es lo mismo hacer un ejercicio que resolver un problema, ya que en el primer caso se aplica un algoritmo, mientras que en el segundo el estudiante debe dar una explicación con respuestas coherentes a las condiciones que plantea, llegando a ser consciente de la responsabilidad que asume en el proceso de enseñanzaaprendizaje (Caseida \& Dávila, 2006). Al respecto, Hidalgo et al. (2015) concluyen que la estrategia del ABP mejora sustancialmente el proceso de aprendizaje de las matemáticas, puesto que evidenciaron que los educandos fortalecieron y mejoraron sus destrezas cuando se enfrentaron a la solución de un problema, generando ambientes más amables a los participantes, dado que el ABP estimuló la investigación tanto grupal como individualmente, haciendo que se profundice y se tenga mejor comprensión de los saberes adquiridos durante el proceso de formación.

De acuerdo con los estudios revisados, se estableció una conexión lógica que sirvió como reflexión inicial para el 
objetivo de la investigación que se describe en este artículo. Se observó que la noción parte-todo con la que generalmente se enseña el número fraccionario, tiene implicaciones que dificultan en los estudiantes la resolución de problemas; y que, tanto docentes como estudiantes, se preocupan por la teoría sin fundamento y de manera memorística, generando en el estudiante la concepción de relevancia únicamente para lo procedimental, alejándolo de una verdadera y consciente disposición de búsqueda de estrategias de solución y dejándolo en el conformismo de ideas pobres y erróneas a cerca de la fracción y sus significados. De ahí que, se considerara como válido un análisis profundo sobre la estrategia didáctica y su implicación en el aprendizaje de la fracción, repensando la forma como se presenta en el aula la fracción y su relación con los diferentes significados y representaciones.

Siguiendo con Silva Laya \& Rodriguez (2011), quien se preocupó por identificar factores en los que también fallan los estudiantes en la resolución de problemas, como lo es la no utilización de saberes previos. Lo cual reafirmó Fuenlabrada et al. (2005), considerando que los estudiantes deben resolver problemas a partir de sus conocimientos anteriores. Es decir, la forma en que el estudiante aborda el problema para resolverlo tiene que ver, además, con la metodología utilizada por el docente.

Continuando con Perera \& Valdemoros (2009), se concluyó que era necesario implementar una estrategia didáctica basada en la resolución de problemas para poder subsanar las fallas que presentaban tanto estudiantes como docentes; puesto que, el abordar los problemas desde los conocimientos previos del estudiante permitió desarrollar la "construcción de significados elementales del concepto fracción"(Pag. 54). Según esto, se podría señalar que el discurso correcto del docente es incomprendido debido a la acumulación de un conocimiento, que se convierte en obstáculo cuando, en aras de un conocimiento nuevo, se aparta totalmente la experiencia del conocimiento anterior y contextualizado por el estudiante.

En síntesis, los estudios mencionados anteriormente, confluyeron en las dificultades que tenían los estudiantes para la resolución de problemas con la fracción y que, en su mayoría, se debían al hecho de obviar los conocimientos previos y a la utilización de una metodología que distaba del contexto. Por ende, la metodología en la aplicación de la fracción y sus significados, fue también objeto de análisis a partir de varios estudios de investigación realizados, en los cuales se identificó el Aprendizaje Basado en Problemas como una de las metodologías utilizada para superar dichas dificultades.

Por todo lo expuesto anteriormente, la aplicación del ABP para el aprendizaje de la fracción y sus significados, es una propuesta válida para que los estudiantes desarrollen Habilidades de comunicación, trabajo en grupo y capacidades de análisis, mientras aprenden a aprender pensando (Vázquez Buenfíl, 2008), identificando
Siguiendo con Silva Laya \& Rodriguez (2011), quien se preocupó por identificar factores en los que también fallan los estudiantes en la resolución de problemas, como lo es la no utilización de saberes previos. 
En la metodología ABP, no se trata de la mera resolución del problema (resultado), sino también la construcción del mismo (proceso). su propio ritmo de aprendizaje desde sus conocimientos previos y la contextualización, en donde la autoevaluación y la coevaluación son parte fundamental del proceso.

\section{Qué es el ABP y cómo funciona}

Teniendo en cuenta la definición de Juárez \& Vizcarro (2008) sobre lo que es el A.B.P y cómo funciona, en principio se requiere que el estudiante apropie una postura particular y argumentada frente a situaciones problema reales, para lo cual no basta enseñar conceptos propios de las disciplinas por simple transmisión, sino que se necesita la práctica pedagógica orientada hacia la búsqueda de nuevos aprendizajes, que requieren de la inmersión del estudiante en el proceso de aprender. Esto significa que los estudiantes descubren sus propios objetivos de aprendizaje enfrentándose a lo que conocen $\mathrm{y} / \mathrm{o}$ desconocen, y profundizando por cuenta propia, desde la interacción que brinda el trabajo en grupo, orientado por el docente y llegando a ser capaces de resolver distintos problemas reales pensando por sí mismos (Polya, 1965). En la metodología ABP, no se trata de la mera resolución del problema (resultado), sino también la construcción del mismo (proceso). Es decir, que el proceso de aprendizaje cobra tanta importancia como los conocimientos adquiridos por el estudiante.

Con base en lo anterior, el ABP funciona a partir de la presentación de una situación problema, siguiendo siete pasos agrupados en tres fases: una discusión previa, estudio independiente y discusión posterior, en la que la socialización es fundamental para que los estudiantes argumenten ideas propias en un ambiente de interacción orientado por el docente, utilizando los aprendizajes específicos como medio y no como un fin en sí mismos; es decir, genera condiciones familiares al pensamiento de los niños.

EntonceselABP,siendounametodología estructurada y definida en diferentes fases, tiene como principal elemento el diseño de situaciones problema para la construcción de nuevos conocimientos, puesto que el diseño debe darse a partir del nivel cognitivo del estudiante y es pertinente al contexto en el que se desea implementar dicha metodología.

\section{Situaciones problema a partir de ABP}

Para poder llegar a implementar la metodología ABP, fue necesario reconocer las características principales a ser tenidas en cuenta en el diseño de situaciones problema como base en la construcción de nuevos conocimientos, para que estas permitan el desarrollo de procesos que promuevan el interés por el conocimiento de conceptos matemáticos a través de la interacción entre docentes y estudiantes, pero también, entre los mismos estudiantes convirtiéndose en un contexto de participación colectiva para el aprendizaje, de manera que el estudiante puede llegar a comprender el significado de conceptos matemáticos, mediante la interacción de este con su deseo por explorar una situación problema dada (Obando Zapata \& Muñera Córdoba, 2003). 
Entonces, Romero \& García (2008) plantean que una situación problema debe ser abierta, sin mostrar las estructuras conceptuales que pretende desarrollar, presentar diversas soluciones en un nivel general $O$ avanzado, y siempre estar amarrada a situaciones reales posibles en sí mismas de ser imaginables. Esto es lo que Streefland (1991) plantea como modelos didácticos que favorecen a los estudiantes en el logro de estadios más elevados de la comprensión, similar a la noción de Vygotsky (1978), en la que la posibilidad de volver a las fuentes debe ser un camino libre y amplio en el que el estudiante avance en el conocimiento matemático, apoyado en la pertinencia del problema ABP. Es decir, el potencial de aprendizaje está dado en la medida en que el estudiante puede volver a un nivel más bajo, aun habiendo alcanzado niveles mayores en la comprensión (Escalona, 2009).

Al respecto, Streefland (1991) indica que la situación problema se ha de desarrollar como un gran reto; pues, los estudiantes lograrán mayores niveles de comprensión solo si dicho problema ha transitado en un crecimiento inter es decir, ha evolucionado en sí misma. Exigencia de ello serán los conceptos matemáticos reconocidos por los estudiantes mediante actividades como planear, identificar, explicar, comparar y plantear soluciones; las cuales han de estar intrínsecas al problema.

\section{La fracción y sus significados como estructura conceptual desde ABP}

Steinbring (1997) resalta la relación que existe entre los signos y los contextos, desde el triángulo semántico que propone en el estudio del significado que tienen los conceptos matemáticos. Con base en lo anterior, en este artículo utilizamos el triángulo semántico como fundamento teórico de porqué el concepto matemático fracción ha de ser estudiado como estructura conceptual desde sus diferentes significados mediante situaciones problema a partir de ABP, reinterpretando el triángulo semántico como el marco de tres elementos fundamentales para este estudio: el concepto fracción, los significados que tiene la fracción y situaciones problema $\mathrm{ABP}$ en las que se encuentra implícito dicho concepto.

Por consiguiente, la fracción se toma como un concepto plurisemántico que desarrolla una estructura conceptual desde y a través de las representaciones: parte todo $\mathrm{a} / \mathrm{b}$, cociente o notación decimal 1.3 , porcentaje $32 \%$ y razón $5: 2$, las cuales dotan de significado al concepto siendo estas el medio de análisis para situaciones naturales al contexto social del estudiante. Dichas representaciones se articulan para el diseño de situaciones problema $\mathrm{ABP}$ que emergen de la realidad en contexto.

\section{Metodología}

El estudio fue realizado bajo el paradigma cualitativo, mediante la investigación acción. Cabe resaltar que las reflexiones contempladas en este artículo son válidas únicamente para el contexto de los niños de séptimo grado de la IEPAGS. De esta manera, se identifica el enfoque crítico social en la medida en que se plantea el interés por estudiar las dificultades que
Esto es lo que Streefland (1991) plantea como modelos didácticos que favorecen a los estudiantes en el logro de estadios más elevados de la comprensión, similar a la noción de Vygotsky (1978), 
En el primer ciclo de investigación, el propósito fue detectar el problema mediante un diagnóstico dividido en tres etapas: Test "Significados y representaciones de la fracción", Taller "Dificultades de análisis en la aplicación de la fracción y sus significados", y cuestionario "percepción de la metodología utilizada". presentan los estudiantes al solucionar problemas que involucran la fracción y sus significados, y que requiere la participación activa de los integrantes de la comunidad estudiada para plantear posibles alternativas de solución, frente a la problemática identificada desde la necesidad de mejorar los procesos de enseñanza y aprendizaje, dejando implícito además la autorreflexión de la propia práctica, tal como lo menciona Popkewitz (1988). En efecto, se abordó dicha problemática desde el contexto propio de los estudiantes y analizó desde el actuar docente, lo que precisamente convoca el tipo de investigación acción (Murillo, 2011) permitiendo al docente ser mediador irreemplazable de la teoría y la práctica (Elliot, 2000).

Todo lo anterior con el propósito de responder al interrogante: ¿Qué efecto tiene la metodología de Aprendizaje basado en problemas en el aprendizaje de la fracción y sus significados?, cuyo objetivo general fue, a través del aprendizaje basado en problemas, desarrollar actividades académicas orientadas al concepto de la fracción y su aplicación, en estudiantes de séptimo grado; con dos objetivos específicos: "Diseñar situaciones problema y aplicarlas con la metodología ABP a estudiantes de séptimo grado para el aprendizaje de la fracción y sus significados" y "Evaluar el efecto del ABP en el aprendizaje de la fracción, sus significados y la solución de problemas".

Cada uno de los objetivos específicos establecidos, fueron anclados a los ciclos de la investigación acción para las cuales se estableció un propósito que permitiera dar cumplimiento a dichos objetivos. Los propósitos fueron validados a través de argumentos teóricos que dan paso a las actividades que se detallan en el diagnóstico y la aplicación de los instrumentos utilizados.

En el primer ciclo de investigación, el propósito fue detectar el problema mediante un diagnóstico dividido en tres etapas: Test "Significados y representaciones de la fracción", Taller "Dificultades de análisis en la aplicación de la fracción y sus significados", y cuestionario "percepción de la metodología utilizada". Cada uno de estos instrumentos de diagnóstico, posteriormente, fueron validados por el juicio de un experto, y tenían como fin medir el grado de interpretación de las diferentes representaciones de la fracción y las relaciones que establecían los estudiantes con los significados del concepto, capacidad del estudiante para plantear soluciones realizando algoritmos y apoyado en sus saberes anteriores, e identificar la percepción de los estudiantes frente a las actividades propuestas dentro del aula y la importancia que daba el docente a cada una de ellas.

En el segundo ciclo elaborar el plan, el propósito fue diseñar situaciones problema a partir de ABP, con base en la temática del curso: Perímetros y áreas, Volúmenes y Estadística. Situación que exigió de las investigadoras, además del conocimiento de la disciplina, creatividad para poder conectar la temática del curso con situaciones reales del entorno y que además estuvieran a nivel cognitivo de la comunidad 
estudiada. Vale la pena mencionar que diseñar una situación problema exigió de tiempo adicional para búsqueda de información, intercambio de ideas con pares, anclar la estructura conceptual que se deseaba profundizar y la relación de esta con otras áreas del conocimiento para poder presentar al estudiante, una situación problema que le permitiera aplicar el concepto fracción y dar sentido a sus significados a través de la realidad.

Seguido a esto, en el tercer ciclo, el propósito fue implementar la metodología ABP mediante las situaciones problema, a dos grupos de estudiantes tomados como muestra, cada una de las situaciones problema para ser resueltas con trabajo colaborativo de cuatro estudiantes por grupo, dando el espacio y tiempo necesario para el desarrollo de las actividades enmarcadas en las tres fases del ABP: discusión previa, estudio independiente y discusión posterior.

Para el ciclo de observación, cuarto ciclo, se tuvo como propósito la sistematización de la información recolectada sobre las actitudes y habilidades desarrolladas y evidenciadas en las tablas de valoración por pares, autovaloración, y soluciones presentadas por los estudiantes.

Para poder analizar dichas actitudes y habilidades, se definieron tres categorías que pretendían dar cuenta de la competencia matemática desde tres aspectos "Trabajo en grupo", "comunicación" y "análisis", respectivamente; las cuales se unieron a tres subcategorías, en su orden:
"Discusión posterior", “discusión previa y socialización” y "estudio independiente". Entonces, las habilidades y actitudes se convierten en indicadores dentro de las subcategorías para ser analizados: resolver el problema, respeto por las ideas de los demás, participación activa en la socialización, leer y analizar el escenario del problema, aporta ideas de tipo académico, definir el problema, realiza trabajo individual, identifica lo que se conoce y no se conoce del problema, formula objetivos de aprendizaje y uso de significados de la fracción. Es decir, cada situación problema permitió medir cada indicador de acuerdo con el diseño cuantitativo realizado para analizar la información recolectada a través de las tablas de valoración por pares, autovaloración y soluciones presentadas por estudiantes.

\section{Resultados}

Un primer acercamiento al problema se hizo desde el diagnóstico, en 3 aspectos diferentes, permitiendo dar una mirada al problema objeto de investigación: primero, "Relación del concepto fracción con sus significados", evidenció que viajar mentalmente desde el concepto a sus significados es un proceso que resulta familiar al estudiante, tienen el concepto y por ahí es fácil encontrar sus significados; pero si el concepto se encuentra implícito en una situación particular, es mucho más complejo relacionarlo desde diferentes significados.

Segundo: "taller de aplicación para identificar las dificultades en la aplicación de la fracción y sus 
significados", mostró que la actitud inicial de los estudiantes fue empezar a realizar operaciones básicas, como: sumas, restas, multiplicaciones y divisiones, con las cuales pretendían dar soluciones a los ejercicios propuestos. Dichas soluciones revelaron análisis pobres y, en la mayoría de los casos, ni siquiera existió comprensión sobre la pregunta del ejercicio. Además, las soluciones planteadas se limitaban a operaciones básicas y dejando de lado aspectos como las unidades de medida que intencionan las magnitudes y que han de ser tenidas en cuenta como eje fundamental para la interpretación del significado de la fracción (Godino \& Batanero, 2002).

Adicionalmente, los estudiantes presentaron problemas de comprensión en la medida fraccional, pues la conceptualización de la fracción no se dio como tal, sino que desde los números naturales los estudiantes contaban tanto las partes que hacían parte de un todo como las partes del todo; es decir, para el estudiante una parte de un todo no podía ser menor a la unidad, entendida esta como una de varias (Obando et al, 2006). Además, los algorítmos que plantearon, hacían referencia a los números naturales sin llegar a comprender cuando se deseaba medir una determinada magnitud en la cual la unidad no estaba contenida un número entero de veces en la magnitud que se quería medir.

Un tercer instrumento de diagnóstico fue el cuestionario con preguntas, con las cuales se pudo evidenciar que para la gran mayoría de los estudiantes, el profesor valoraba más la respuesta que el procedimiento. En relación con lo anterior, podemos reinterpretar a Kamii (1994), cuando se refiere a la necesidad que el docente permita que los estudiantes construyan las estructuras conceptuales de los conceptos matemáticos, paso a paso, desde sus conocimientos anteriores, y no desde ejercicios en los que se aplican las fórmulas que generalizan el concepto desde situaciones concretas o ejemplos, y que luego tendrán que ser mecanizadas y replicadas por el estudiante para evidenciar que ha "aprendido" (Obando et al, 2006).

Entonces, los estudiantes manifestaban que la metodología utilizada con ellos apuntaba hacia el desarrollo de ejercicios modelo, en los cuales se les valora únicamente la respuesta desconociendo el camino que el estudiante ha transitado para llegar a ella, como lo evidencia el mismo cuestionario en el que los estudiantes expresaron que la mayoría de actividades propuestas por el docente en el aula correspondían a talleres que desconocen las características del trabajo en equipo y desligan procesos de análisis y comprensión, los cuales quedaron en último lugar de las actividades propuestas en el aula, y que, según los estudiantes, el docente consideraba relevantes para lograr los aprendizajes, dando mucha mayor trascendencia al respeto y la disciplina, lo que dejó entrever un sistema tradicional de clase magistral impartida por el docente.

Por tanto, los estudiantes expresaron que las actividades encaminadas al análisis, a valorar cómo aprenden y potencian los conocimientos acumulados en años 
anteriores de estudio, se han dejado de lado, constituyendo la enseñanza en la solución de algorítmos con respuestas correctas, como un molde en el que todos han de caber, haciéndose menos competentes y en el que tanto docentes como estudiantes se niegan la posibilidad de enseñar y aprender a pensar (Kamii, 1994).

Una vez realizado el diagnóstico e identificada la problemática, se pensó la enseñanza de la fracción y sus significados en concordancia con el MEN (2006) y los derechos básicos de aprendizaje para grado séptimo, que definen la competencia a desarrollar como la resolución de problemas utilizando la fracción y sus significados y mediante una propuesta didáctica basada en la solución de problemas (con las actitudes y habilidades que deben ponerse en acción), la cual implicó que los instrumentos y criterios a valorar no fueran los tradicionales, pues en este punto pierde protagonismo el limitarse a cotejar el resultado obtenido con el correspondiente a una operación y cobra importancia la consideración del proceso de acción y de las actitudes con respecto a proponer soluciones y atender las propuestas de otros. Lo cual fue el punto de partida para elaborar el diseño cuantitativo y medir los indicadores, según la valoración de los ítems establecidos en las tablas de valoración diligenciadas por cada estudiante en cada una de las situaciones problema.

La evaluación de los ítems se realizó teniendo en cuenta el Índice Sintético de Calidad Educativa (ISCE) en su componente Progreso, de manera que se asemejó el nivel insuficiente con el criterio de valoración "no puede hacerlo"; y el nivel avanzado, con el criterio de valoración "seguro de hacerlo". Es decir, a más estudiantes en "no puede hacerlo" y más estudiantes en "seguro de hacerlo", se consideró el indicador como una debilidad; a menos estudiantes en "no puede hacerlo" y menos estudiantes en "seguro de hacerlo", se consideró oportunidad; a menos estudiantes en "no puede hacerlo" y más en "seguro de hacerlo", se consideró fortaleza; y más estudiantes en "no puede hacerlo" y menos estudiantes en "seguro de hacerlo", se consideró amenaza.

Por lo tanto, las categorías se catalogaron de acuerdo con el número de ítems que cada indicador tuvo en debilidades, oportunidades, fortalezas y amenazas. Se encontró que indicadores como: Presenta la información organizada, interviene en la solución del problema, expresa con claridad sus puntos de vista, comprende la información que expone e identifica sus necesidades de aprendizaje, entre otros, se ubicaron como oportunidades y fortalezas, dejando muy poco en debilidades y amenazas. Dentro de las amenazas, se ubicó el uso de la fracción como razón, siendo esta la principal dificultad encontrada en el diagnóstico, lo que sugiere que al respecto es importante seguir profundizando en la aplicación de la fracción dentro de contextos que así la requieran.

\section{Conclusiones}

La intervención en el aula realizada con los estudiantes de séptimo grado de la IEPAGS, dio oportunidad para identificar amenazas y debilidades
Dentro de las amenazas, se ubicó el uso de la fracción como razón, siendo esta la principal dificultad encontrada en el diagnósti$\mathrm{co}$, lo que sugiere que al respecto es importante seguir profundizando en la aplicación de la fracción dentro de contextos que así la requieran. 
del proceso, pero también valiosas oportunidades y fortalezas que los estudiantes tienen y que con otra metodología serían muy difícil de identificar. Además, la forma de valoración formativa del proceso, se convierte en elemento fundamental para lograr analizar los resultados, puesto que no se precisa el estudiante como un número sino que es evaluado a lo largo del proceso en sus habilidades, es decir, se reconoce el interés del estudiante por indagar más en las temáticas propuestas, el desarrollo de habilidades comunicativas que propician una interacción en convivencia, lo cual visto desde el ISCE se convierte en un aporte para el componente "ambiente escolar", mejorando el clima de aula y capacidades de análisis que contribuyen concretamente en el desarrollo del pensamiento matemático y, por ende, en el desempeño de los estudiantes en situaciones problema de la vida real.

En conclusión, la metodología ABP para el aprendizaje de la fracción en grado séptimo de los niños de la IEPAGS, se cataloga como una fortaleza desde el análisis y como una oportunidad en cuanto al trabajo en grupo y la comunicación. Es decir, abre la posibilidad de explorar y desarrollar las habilidades comunicativas, de trabajo en grupo para que los estudiantes sean más competentes en la resolución de problemas, y muestra que definitivamente logra fortalecer en los estudiantes las capacidades de análisis frente a situaciones problema particulares.

\section{Referencias}

Angulo, M., \& Castaño, O. (2011). Actividades didácticas en enseñanza secundaria para el desarrollo de pensamiento aleatorio. Scientia Et Technica, 16(49), 158162.

CASEIDA, C., \& DÁvila, E. (2006). El aprendizaje basado en problemas y proyectos: Una estrategia de integración. Estado libre asociado de Puerto Rico: Departamento de educación Universidad de educación Interamericana de Puerto Rico, recinto de Bayamon.

Castaño, N. (2014). Dificultades en la enseñanza de las operaciones con números racionales en la educación secundaria. Recuperado de: http:// repositorio.autonoma.edu.co/jspui/bitstream/11182/861/1/Tesis\%20 N\%C3\%A9stor $\% 20$ Mario $\% 20$ Casta $\%$ C3\%B1o.pdf

DiAz, D. (2010). Sistema de ecuaciones y resolución de problemas: una propuesta de enseñanza y aprendizaje. Recuperado de http://funes.uniandes.edu. co/4991/1/CB_39.pdf

Elliot, J. (2000). El cambio educativo desde la investigación acción. Madrid: 
Ediciones Morata S.L.

Escalona, H. (2009). Correo del maestro. Recuperado de http://www. correodelmaestro.com/

Escolano, R., \& Gairín, J. M. (2005). Modelos de medida para la enseñanza del número racional en educación primaria. Revista Iberoamericana de educación matemática, (1), 17-35.

Fuenlabrada, I: et al. (2005). Aprender a enseñar matemáticas. Monterrey: Centro de altos estudios e investigación pedagógica.

Godino, J. D., \& Batanero, C. (2002). Proporcionalidad y su didáctica para maestros. Granada: Departamento de Didáctica de la Matemática.

Hidalgo, P. H: et al. (2015). Aprendizaje basado en problemas como potencializador del pensamiento matemático. Plumilla Educativa, (15), 299-312. Recuperado http://revistasum.umanizales.edu.co/ojs/index.php/plumillaeducativa/ article/view/845/2698

Juárez, E., \& Vizcarro, C. (2008). Qué es el ABP y cómo funciona? Madrid: Universidad de Murcia.

KAMII, C. (1994). Reinventando la aritmética III. Madrid: Visor.

MEN. (2016). Ministerio de educación. Recuperado de mineducación.gov.co

Morales Díaz, R. O. (2014). Dificultades y errores en la solución de problemas con números racionales. Recuperado de: http://repositorio.autonoma.edu.co/ jspui/bitstream/11182/865/1/Informe\%20final\%20Raul\%20Morales $\% 20$ con $\% 20$ toda $\% 201 \mathrm{a} \% 20$ bibliografia $\% 20$ diembre $\% 20$ toda $\% 20$ completa.pdf

Murillo, T. F. (2011). Investigación-acción. Madrid: stmaria.

Obando Zapata, G. \& Muñera Córdoba, J. (2003). Las situaciones problema como estrategia para la conceptualización matemática. Revista Educación y Pedagogía, 15 (35), 185-199.

Obando, Z. G. et al. (2006). Pensamientos Numéricos y Sistemas Numéricos. Medellín, Colombia: Artes Y letras LTDA.

Perera, P., \& Valdemoros, M. (2009). Enseñanza experimental de las fracciones en cuarto grado. Educación matemática, 21, 29-61. Recuperado de https:// dialnet.unirioja.es/servlet/articulo?codigo $=2697033$

Polya, G. (1965). Cómo plantear y resolver problemas. México: Trillas.

Popkewitz, T. (1988). Paradígma e ideología en investigación educativa. Las funciones sociales del intelectual. Madrid: Mondadori.

Romero, M. A., \& García, S. J. (2008). La elaboración de problemas A.B.P. En U. d. Murcia, La metodología del A.B.P. Madrid: Universidad de Murcia.

Silva Laya, M., \& Rodriguez, F. A. (2011). ¿Por qué fallan los alumnos al resolver problemas matemáticos? Didáctica, enseñanza y aprendizaje de las 
matemáticas, (56), 21-28

SteINBRING, H. (1997). Epistemological investigation of classroom interaction in elementary mathematics teaching. Educational Studies in Mathemattics,32(1), 49-92.

Streefland, L. (1991). Fractions in realistic mathematics education. Kluwer Academic Publishers, 46-134.

VÁzQuez Buenfíl, A. (2008). Consideraciones sobre el aprendizaje basado en problemas (ABP) en matemáticas. Facultad de educación de la universidad Autónoma de Yucatán, 11(35), 73-83.

Vygotsky, L. (1978). Mind and society. The development of higher psychological processes. Cambridge, massachusettes: Harvard University Press. 